\title{
Comment on: "Intracytoplasmic morphologically selected sperm injection (IMSI) does not improve outcome in patients with two successive IVF-ICSI failures" by Gatimel et al.
}

\author{
K. Lukaszuk ${ }^{1,2} \cdot$ E. Pastuszek ${ }^{1,2} \cdot$ A. Samojedny ${ }^{1}$
}

Received: 18 May 2016 / Accepted: 19 May 2016/Published online: 30 May 2016

(C) The Author(s) 2016. This article is published with open access at Springerlink.com

Dear Editor,

We have read with great interest the article "Intracytoplasmic morphologically selected sperm injection (IMSI) does not improve outcome in patients with two successive IVF-ICSI failures" by Gatimel and colleagues [1].

However, we have to bring attention to the problem which is unfortunately repeated in many articles and shows the weakness of our field in the application of basic physics. The authors state that the spermatozoa were observed under $\times 6000$ magnification. It is a very misleading claim that makes it seem that it is indeed possible to obtain such magnification using a standard optical microscope (even a most advanced one). Calculation of the actual real magnification is impossible as the article does not list the basic microscope parametersobjective numeric aperture (NA), condenser NA and wavelength that was used to observe the spermatozoa $[2,3]$.

Nevertheless, let us try to calculate the actual real magnification used by the authors. We know that the objective's magnification was $\times 100$. It is described as an immersion microscope so we can assume the immersion was in oil (as opposed to air). The objective was mounted on the Leica DMI 6000 inverted microscope. Available objectives of this type have the NA from 1.3 to 1.49. Let's assume the NA of 1.4.

Resolution, and consequently the real magnification, also depends on the condenser. As no specific information is provided,

E. Pastuszek

ewa_pastuszek@gumed.edu.pl

1 INVICTA Fertility and Reproductive Center, Wroclaw Gdansk, Warsaw, Poland

2 Department of Obstetrics and Gynecological Nursing, Faculty of Health Sciences Medical, University of Gdansk, Gdansk, Poland we could deduce that, in the best case scenario, the microscope had the condenser typically used in cell cultures and IVF laboratories. These kinds of condensers need to have a relatively large working distance that always means a lower condenser NA.

A condenser allowing moderate comfort of work that could be used with a $\times 100$ objective would be TI-C-LWD LWD (numerical aperture 0.52).

Wavelength is another parameter that has not been provided. In order to get optimal resolution, a filer was probably used. We can assume the use of a standard filter, i.e., a green $550-\mathrm{nm}$ filter. Resolution can be calculated based on the following formula:

$$
r=\frac{1.22 \lambda}{N A_{\mathrm{obj} .}+N A_{\mathrm{cond} .}}
$$

In a properly configured microscope: $N A_{\mathrm{obj}}{ }^{+}$ $N A_{\text {cond. }}=2 N A_{\text {obj. }}$.

We arrive at $0.35 \mu \mathrm{m}$. As the resolution of the human eye is approximately $300 \mathrm{dpi}$ (dots per pixel) or $83 \mu \mathrm{m}$ pixels on the screen $30 \mathrm{~cm}$ away from the human eye, we arrive at the magnification of $\times 237$.

If we assume a much worse than usual eyesight (100 dpi) with the use of an ideal objective with NA of 1.49, T-C High NA condenser with NA of 0.85 , and a $400-\mathrm{nm}$ filter (blue filter that gives the highest resolution achievable with the human eye), we will get the real resolution of $0.21 \mu \mathrm{m}$ and real magnification of only $\times 1199$.

We can say, simplifying it greatly, that the real magnification of a microscope can be most easily estimated by multiplying the objective's NA by 1000 . It is a highly idealized model as exemplified by above calculations.

This should all be taken into account when information about microscopes is included in publications and when used to rationalize data and results. 
Open Access This article is distributed under the terms of the Creative Commons Attribution 4.0 International License (http:// creativecommons.org/licenses/by/4.0/), which permits unrestricted use, distribution, and reproduction in any medium, provided you give appropriate credit to the original author(s) and the source, provide a link to the Creative Commons license, and indicate if changes were made.

\section{References}

1. Gatimel N, Parinaud J, Leandri RD. Intracytoplasmic morphologically selected sperm injection (IMSI) does not improve outcome in patients with two successive IVF-ICSI failures. J Assist Reprod Genet. 2016;33:349-55.
2. James J, Tanke HJ. Biomedical light microscopy, softcover reprint of the original 1st ed. 1991st ed. New York: Springer Science \& Business Media; 2012.

3. Spencer M. Fundamentals of light microscopy (IUPAB Biophysics Series). 1st ed. Cambridge: Cambridge University Press; 1982. 